\title{
Rethinking Maternal Sensitivity: Mothers' Comments on Infants' Mental Processes Predict Security of Attachment at 12 Months
}

\author{
Elizabeth Meins, Charles Fernyhough, Emma Fradley, and Michelle Tuckey \\ Staffordshire University, Stoke-on-Trent, U.K.
}

\begin{abstract}
This study investigated predictors of attachment security in a play context using a sample of 71 mothers and their 6-month-old infants. We sought to rethink the concept of maternal sensitivity by focusing on mothers' ability accurately to read the mental states governing infant behaviour. Five categories were devised to assess this ability, four of which were dependent on maternal responses to infant behaviours, such as object-directed activity. The fifth, mothers' Appropriate mind-related comments, assessed individual differences in mothers' proclivity to comment appropriately on their infants' mental states and processes. Higher scores in this fifth category related to a secure attachment relationship at 12 months. Maternal sensitivity and Appropriate mind-related comments were independent predictors of attachment security at 12 months, respectively accounting for $6.5 \%$ and $12.7 \%$ of its variance. We suggest that these findings are in line with current theorising on internal working models of attachment, and may help to explain security-related differences in mentalising abilities.
\end{abstract}

Keywords: Attachment, individual differences, mothers, parent-child interaction, normal development, mind-mindedness.

Abbreviations: AAI: Adult Attachment Interview; IWM: internal working model.

\section{Introduction}

Since the seminal work of Ainsworth and colleagues (Ainsworth, Bell, \& Stayton, 1971, 1974; Ainsworth, Blehar, Waters, \& Wall, 1978), there have been a number of attempts to identify the antecedents of infantile security of attachment. Of the many aspects of early infantmother interaction that have been investigated as possible precursors of the secure attachment relationship, there has been some consensus that a key variable may be sensitive mothering in the first year of life. In their pioneering study, Ainsworth et al. (1971) established four dimensions for assessing maternal behaviour in early infant-mother interactions: sensitivity, acceptance, cooperation, and accessibility. They reported that infants of mothers who demonstrated higher levels of sensitivity were more likely to show secure attachment behaviour in the Strange Situation at 12 months, with mothers who scored highly on sensitivity also showing more acceptance, cooperation, and accessibility in their interactions with their infants.

Subsequent researchers have found broad support for the relation between early maternal sensitivity and the security of the attachment relationship. For example, several studies employing Ainsworth et al.'s sensitivity scale have replicated the original findings, not just in comparable American samples (e.g. Isabella, 1993), but also in German infant-mother dyads (Grossmann, Grossmann, Spangler, Suess, \& Unzner, 1985) and in

Requests for reprints to: Dr Elizabeth Meins, Department of Psychology, University of Durham, Science Laboratories, South Road, Durham DH1 3LE, U.K.

(E-mail: elizabeth.meins@durham.ac.uk). high-risk populations (Egeland \& Farber, 1984; Goldberg, Perotta, Minde, \& Corter, 1986). The quality of early infant-mother interaction has also been assessed using indices of maternal behaviour such as interactional synchrony (Isabella, Belsky, \& Von Eye, 1989) and harmony (Schölmerich, Fracasso, Lamb, \& Broberg, 1995), and a measure of the extent to which the mother stimulates the infant (Belsky, Rovine, \& Taylor, 1984). Although not directly assessing maternal sensitivity, these latter studies have shown that mothers demonstrating greater attunement to their infants in these areas of measurement were more likely to have securely attached infants, a fact that has been interpreted as further evidence for the link between sensitive mothering and security. At the same time, the conclusion that maternal sensitivity is the key determinant of attachment security has not been left unchallenged. For example, the results of a recent meta-analysis on the parental antecedents of infant attachment (De Wolff \& Van IJzendoorn, 1997) raised doubts about whether the relation between sensitivity and security was as strong as Ainsworth's original findings led one to believe. This meta-analysis also highlighted a wide range of parental behaviours that were related to attachment security, leading De Wolff and Van IJzendoorn (1997) to conclude that "[s]ensitivity cannot be considered to be the exclusive and most important factor in the development of attachment" (p. 585).

The resulting picture of maternal sensitivity as a determinant of attachment security is thus a somewhat confused one. We suggest that this confusion may have arisen for a number of reasons: (1) the rather general and coarse-grained nature of Ainsworth et al.'s original maternal sensitivity scale; (2) a lack of consensus among subsequent researchers concerning which behaviours are taken to constitute maternal sensitivity; and (3) the 
failure of research in this area consistently to consider maternal behaviour in light of its interactional context. As we shall argue, the central criterion of Ainsworth et al.'s original definition of sensitivity is that the mothers' response to the infant is not merely prompt or contingent, but appropriate to the infant's behaviour. This feature of maternal behaviour, which in Ainsworth et al.'s theorising is crucial to the establishment of a secure attachment relationship, has been increasingly overlooked by recent research. We suggest that a rethinking of the concept of maternal sensitivity, which goes some way to take into account the appropriateness of maternal responses to their infants, might allow us to clear up some of the confusion surrounding this important concept, and thus make possible an investigation of the antecedents of attachment security that is more in line with Ainsworth et al.'s original insights.

\section{Defining Maternal Sensitivity}

Ainsworth et al. (1971) described the mother of a securely attached child as being "capable of perceiving things from [the child's] point of view" and regarding her child "as a separate person; she also respects his activityin-progress and thus avoids interrupting him" (p. 43). This definition suggests that the distinguishing feature of such mothers is their ability to use information from their children's outward behaviour in making accurate inferences about the mental states governing that behaviour. This feature of maternal cognition would thus appear to go beyond a basic ability merely to recognise and respond to the child's physical states, such as hunger, and emotional states, such as distress. Ainsworth et al. reported that, compared with their secure group counterparts, insecure group mothers appeared less able to "read" their infants' behaviour, leading them to try to "socialize with the baby when he is hungry, play with him when he is tired, and feed him when he is trying to initiate social interaction" (Ainsworth et al., 1974, p. 129). What distinguishes insecure group mothers is thus not a general failure to respond to their children; rather their responses are more likely to be inappropriate because they are less able or willing to evaluate why the child is demonstrating a particular behaviour.

Unfortunately, much of the research on maternal behavioural antecedents of attachment in the interim has paid insufficient attention to Ainsworth et al.'s distinction between mothers' responsiveness to infants' emotional cues and the appropriateness of each response. For example, over half of the 68 studies included by De Wolff and Van IJzendoorn (1997) in their meta-analysis focused exclusively on the contiguity or synchrony of responses, or on maternal stimulation or support, without any assessment of whether a mother's responses were appropriate to cues from the child. Indeed, even when researchers have measured sensitivity using Ainsworth et al.'s (1971) scale, a lack of specificity in the original operationalisation of the construct has led inevitably to differing interpretations of the criteria for sensitive mothering. Ainsworth et al.'s scale is a global measure based on the observer's perception of the mother's sensitivity throughout the observation period; each mother receives a score between 1 and 9, with higher scores indicating greater sensitivity. Observers are not given guidelines about the specific behaviours that should be coded, or whether the frequency with which behaviours occur is important; the individual researcher may also choose the length and structure of the observational period. This lack of specificity in the original scale may help to explain the general failure to replicate the very strong relation between sensitivity and attachment security reported by Ainsworth et al. (1978) (see De Wolff \& Van IJzendoorn, 1997; Goldsmith \& Alansky, 1987).

\section{Observational Contexts}

As well as considering the appropriateness of maternal responses to their children, it is also important that researchers pay full attention to the context within which such behaviours occur. Ainsworth et al. (1978) concluded that "the most important aspect of maternal behaviour commonly associated with the security-anxiety dimension of infant attachment is manifested in different specific ways in different situations, but in each it emerges as sensitive responsiveness to infant signals and communications" (p. 152, emphasis added). For example, if one observes infant-mother interaction in a situation that is likely to provoke distress, the most appropriate demonstration of sensitivity will be for the mother promptly to offer comfort and reassurance. If one observes feeding routines, then sensitivity will be manifested in the mothers' attunement to the infant's cues of hunger and satiety, and the tempo at which the infant wishes to feed. However, when the child is physically and emotionally satisfied, the most appropriate kind of sensitivity would appear to be the mothers' reading of her infant's focus of attention, readiness to play, and enjoyment of particular kinds of activity. The latter type of sensitivity - that demonstrated when the child is physically and emotionally satisfied - is clearly a manifestation of the mother's sensitivity to her infant's mental state. Indeed, we suggest that this capacity to "[perceive] things from [the child's] point of view" (Ainsworth et al., 1971, p. 43) provides the key to a successful rethinking of the concept of maternal sensitivity.

\section{Rethinking Maternal Sensitivity}

In response to the rather confused picture of maternal sensitivity that has emerged since Ainsworth et al.'s (1971) original findings, researchers in this area have begun to call for a re-examination of this important concept. In particular, Fonagy, Steele, Steele, Higgitt, and Target (1994) and Meins (1997) have argued that responsiveness to the child's physical and emotional needs should be clearly distinguished from mothers' capacity or willingness to engage with their infants at a mental level. Using new theoretical constructs, Fonagy et al. and Meins have hypothesised that maternal behaviours that betray a sensitivity to infants' mental states, rather than responsivity to their physical and emotional needs, will be more useful than a generalised construct of maternal sensitivity in predicting the security of the attachment relationship.

To this end, Meins (1997) coined the term maternal mind-mindedness to describe the mother's proclivity to treat her infant as an individual with a mind, rather than merely as a creature with needs that must be satisfied. The concept of mind-mindedness clearly captures the flavour of Ainsworth et al.'s distinction between sensitive and insensitive mothering. That is, the mind-minded mother is sensitive to the child's "work-in-progress", is willing to change her focus of attention in response to cues from the infant, and so on. 
As an alternative to Ainsworth et al.'s original construct of maternal sensitivity, the construct of mindmindedness allows us to distinguish between a mother's general sensitivity to her child's physical and emotional needs (suggested by Ainsworth et al., 1974, to be a feature of both secure- and insecure-group mothers), and a more specific sensitivity to the child's mental states and ongoing activity. This ability to "read" the mental states underlying a child's behaviour is most likely to be apparent in situations where the child's physical and emotional needs are satisfied. In contexts such as free play, mind-minded mothers will be able to respond to behaviours such as their infants' current level of engagement in joint activities, shifts of attention, etc. in a manner that is unconstrained by the requirements of caregiving. Inasmuch as they capture the essential quality of maternal sensitivity as defined by Ainsworth et al., individual differences in such behaviour will in turn be expected to relate to the subsequent security of the attachment relationship, with infants of mothers with higher levels of mind-mindedness being more likely to be classified as securely attached.

Meins and colleagues have already investigated maternal mind-mindedness and its relation to security of attachment within the contexts of mothers' descriptions of their children, and their interpretations of their children's vocabulary items at 20 months. Meins, Fernyhough, Russell, and Clark-Carter (1998) reported a link between security of attachment in infancy and mothers' descriptions of their children at age 3 years. In comparison to their insecure group counterparts, mothers whose infants had been securely attached were more likely to focus on their mental characteristics, rather than their physical appearance or behavioural tendencies, when given an open-ended invitation to describe their children 2 years later. In addition, Meins (1998) found that secure group mothers were more willing or able to attribute meaning to their children's early vocalisations by, for example, maintaining that certain utterances that did not conform to actual English words were nevertheless being used systematically by their children to convey a specific meaning. Such proclivities in the secure group mothers were interpreted as evidence for their greater mind-mindedness. Other researchers (Beeghly, Bretherton, \& Mervis, 1986; Dunn, Bretherton, \& Munn, 1987) have noted individual differences in the frequency with which mothers use mental state language when interacting with their children during the second and third years of life. Both Beeghly et al. (1986) and Dunn et al. (1987) also reported that, as children get older, their mothers increasingly refer to internal states, such as cognitions, emotions, and obligations. Previous research has not, however, investigated whether such differences in mind-minded psychological discourse exist in mothers' interactions with their very young infants, and thus predate the formation of the attachment relationship. Extending the scope of the existing concept of mindmindedness to evaluate its role as a predictor, rather than a consequence, of attachment security is the focus of this paper.

Our general aim is thus to attempt a rethinking of maternal sensitivity in terms of mothers' specific proclivity to focus on and respond to their infants' mental states, as manifested in their ongoing behaviour. In so doing, we hope to refine the concept of maternal sensitivity in a way that is more in line with Ainsworth et al.'s original insights. Any new concept that results from such a rethinking must, however, be at least as good a determinant of subsequent patterns of attachment as maternal sensitivity. For example, it will be necessary to demonstrate that maternal mind-mindedness can not only distinguish between infants using the dichotomous secure and insecure categories, but also between infants in the avoidant and resistant insecure groups. In terms of sensitivity, mothers of insecure-avoidant infants are the least sensitive of the three original attachment categories, tending to reject their infants' bids for attention and interaction (Ainsworth et al., 1978; Main, 1981). Mothers of insecure-resistant infants have been found to be inconsistent in their patterns of mothering, sometimes demonstrating high levels of sensitivity and at other times being insensitive to their infants' needs (Isabella, 1993). Thus, in order to represent a progression in understanding the antecedents of attachment security, any new construct will similarly need to be able to distinguish between the insecure groups.

The study reported below involved assessing maternal behaviour in an interactional play context on scales of both maternal mind-mindedness and maternal sensitivity, in order to investigate their relative power in predicting subsequent security of attachment. The initial goal was to establish empirical measures for assessing maternal mindmindedness which were appropriate to the age of the infants studied (6 months) and to the interactional context. This in turn allowed us: (1) to investigate the relation between maternal mind-mindedness and Ainsworth et al.'s construct of maternal sensitivity; (2) to attempt to replicate the previously observed relation between maternal sensitivity and subsequent security of attachment; (3) to test the hypothesis that infants whose mothers show higher levels of mind-mindedness at 6 months will be more likely to be securely attached at 12 months; (4) to determine the relative contribution of maternal sensitivity and mind-mindedness to children's subsequent security of attachment; and (5) to investigate whether differences in maternal mind-mindedness could distinguish between infants in the avoidant and resistant insecure attachment categories.

\section{Method}

\section{Participants}

Participants were 71 pairs of mothers and infants ( 36 boys, 35 girls) who were first seen when the infants were 6 months of age ( $M=25$ weeks; range: $23-28$ weeks). The average age of the mothers at the beginning of the study was 28 years (range: 19 42 years) and the participating families were predominantly lower-middle class. The sample was recruited via local health centres and baby clinics, and $60 \%$ of mothers who were approached agreed to take part ${ }^{1}$. Mothers and infants were followed up at 12 months ( $M=53$ weeks; range: 52-56 weeks).

${ }^{1}$ The percentage of mothers agreeing to participate is somewhat low. We attribute this to the fact that the local inhabitants were unused to being approached by university researchers seeking participants for their projects. Rather than being a university town, the catchment area for the study consisted of a large conurbation of towns in an industrial area of the English Midlands, and this study was the first on infant psychological development to be carried out using this population. Mothers who did not wish to participate were not asked directly to give specific reasons for refusing, but in the vast majority of cases mothers explained that refusal resulted from time constraints and pressures. The information available on mothers who refused gave no reason to suggest that they differed in any significant way from those who agreed to participate. 


\section{Maternal Education}

Mothers' level of education was included as an independent variable, since it was necessary to control for the possibility that aspects of infant-mother interaction, and particularly mindmindedness, may relate to the amount of time mothers have spent in the education system. Given the focus of this study, maternal education was therefore deemed to be a more appropriate control variable than a general measure of socioeconomic status. Mothers were given a questionnaire in which they were asked to identify their highest educational qualification by choosing one of six categories. Each mother was awarded one of the following scores for educational level ${ }^{2}: 0$ : no examinations; 1 : CSEs (equivalent to high school up to age 16 for less academic students); 2: GCSEs or O-Levels (high school up to age 16 for more academic students); 3: A-Levels (high school up to age 18); 4 : further qualification, not to degree level (e.g. nursing); 5: undergraduate degree; 6: postgraduate qualification. Of the 71 mothers taking part: 7 scored $0 ; 7$ scored $1 ; 22$ scored $2 ; 3$ scored $3 ; 23$ scored $4 ; 5$ scored 5 ; and 3 scored 6 .

\section{Phase One Testing (Age 6 Months)}

The testing session at 6 months of age was carried out in the University's developmental research laboratory. Mothers had been sent a letter explaining the time course of the study, and detailing what was required of them at each testing phase. However, participants were not explicitly told that the study was assessing infant-mother attachment and investigating ways in which mothers could demonstrate their mind-mindedness. Mothers and infants were introduced into the testing room, in which there were several easy chairs, three large floor cushions, and a range of age-appropriate toys. Mothers were told that they could move around the room as they wished as the session progressed, but all sessions began with the mother and infant sitting on the floor cushions in the middle of the room. No specific instructions were given, other than an invitation to each mother to play with her baby as she would do if they had a few spare minutes together at home.

The mother and infant were allowed a 5-minute settling-in period before the video recording began. There were two video cameras, mounted on the walls in diagonal corners of the room. The video mixing desk allowed a split-screen representation, so that the faces of both the mother and infant could be clearly seen at all times. The first 20 minutes of videotape from these sessions was used in the analyses.

\section{Phase One Scoring Procedure}

The infant-mother interactions at the 6-month testing session were coded for a range of maternal and infant behaviours.

Infant behaviours. Three types of infant behaviour were coded:

(1) Infant vocalisations: any vocal noise made by the infant. A gap of at least 2 seconds between discrete vocalisations was required for them to be counted separately.

(2) Infant changes in direction of gaze, where a "gaze" was defined as any look of 2 or more seconds in duration.

(3) Infant object-directed actions: any action directed toward an object, such as touching, grasping, fingering, pointing.

Infants received a frequency score for each of these categories. The videotaped interactions were coded by a trained researcher, and a randomly chosen fifth of these tapes was coded by a second researcher, both of whom were blind to the measures of

\footnotetext{
${ }^{2}$ North American equivalents to the British educational system
} are shown in parentheses. maternal sensitivity, security of attachment, and infants' general cognitive ability. Inter-rater agreement was $\kappa=.86$.

Maternal behaviours. Mothers' behaviour was coded for: (1) sensitivity; and (2) mind-mindedness.

(1) Maternal sensitivity. The sensitivity of mothers' interactions with their infants was coded using Ainsworth et al.'s (1971) scale. This is a 9-point scale, with 5 "anchor points" (highly sensitive, sensitive, inconsistently sensitive, insensitive, highly insensitive). Rather than coding specific behaviours, this scale gives a global rating of how sensitive the mother is to the infant's cues. The coder was therefore not required to score or assess the minutiae of the interactions, but rather assessed each mother's overall sensitivity during the course of the whole 20minute session. Higher scores on this scale are indicative of more sensitive mothering. The videotaped sessions were coded by a trained researcher, and a fifth of the tapes chosen at random were coded by a second researcher. Both researchers were blind to all of the other measures. Using the 9-point scale, inter-rater agreement was $\kappa=.75$, with exact agreement for $79 \%$ of the observations.

(2) Mind-mindedness. In order to devise dimensions for measuring maternal mind-mindedness, the first two authors made detailed observations of the videotaped interactions of six infant-mother pairs who were chosen at random. The development of the coding system involved determining ways in which a mother could demonstrate that she was treating her infant as a mental agent, capable of intentional action. In order for a behaviour to become established as a category of mindmindedness, all of the six mothers had to demonstrate this behaviour at least once during the 20-minute session. Five measures of mind-mindedness were identified: Maternal responsiveness to change in infant's direction of gaze; Maternal responsiveness to infant's object-directed action; Imitation; Encouragement of autonomy; and Appropriate mind-related comments. These categories are described in detail below.

The two researchers who coded the infant behaviours categories (see above) also coded the videotapes for the maternal mind-mindedness categories. Every vocal comment the mother made was categorised. A maternal vocal comment was defined as a discrete sound or single word, or at the level of a sentence in longer utterances. For example, mothers often made single word comments which served to label some aspect of the environment, and would repeat this word several times, e.g. "Teddy. Teddy". This utterance would be scored as two separate comments. Mothers thus received scores for the total number of vocal interjections made during the 20-minute episode.

Obviously, the mind-mindedness categories were not exhaustive of all types of maternal behaviours observed during the free play sessions. Therefore, in addition to the mind-mindedness categories, there was a miscellaneous Other category, and any maternal behaviours the coders deemed not to be mind-minded were placed into this category. The Other category included comments giving positive or negative feedback on the infant's behaviour, behaviours which served to direct the infant's attention, and engagement in standardised game routines, such as "peekaboo". Inter-rater agreement for assignment of maternal behaviours across the five mind-mindedness categories and the Other category was $\kappa=.90$.

One way in which mothers appeared to demonstrate their mind-mindedness was in their responses to the three Infant behaviour categories described above. The first two categories of mind-mindedness emerged from the ways in which mothers responded to subtle infant behaviours which could be interpreted as cues for engagement in or disengagement from activities. Infants' line of gaze and object-directed action appeared to function as this type of cue, giving the categories Maternal responsiveness to change in infant's direction of gaze and Maternal responsiveness to infant's object-directed action.

For these two categories, maternal responsiveness included mothers looking at the object on which the infant's attention was focused, touching or picking the object up, or naming or describing the event or object to which the infant was directing 
behaviour. Scores for both of these categories were proportional. For the first category, each mother received a score for the number of changes in attention she made in response to her infant's line of gaze as a proportion of the total number of gaze changes made by her infant (see Infant behaviours above). For the second category, each mother received a score for the number of changes in attention she made to the infant's objectdirected action as a proportion of the total number of objectdirected actions performed by her infant (see Infant behaviours above). These proportional scores, based on the contingency of maternal responses, were used in the analyses. Mothers' responses in such situations were deemed to be indicative of mind-mindedness since they provided an assessment of each mother's capacity to recognise that her infant's agenda was not always the same as her own. Mothers who are sensitive to their infants' mental life will be more likely to use the infant's change of attention as a cue to centre their own behaviour on the infant's new focus of interest. In contrast, a less mind-minded mother might ignore such changes in attention, attempt to focus the infant back on the activity in which she herself is engaged, or simply fail to register that her infant is no longer paying attention. These two categories were dependent on the infant's behaviour and can thus be seen as a measure of maternal responsiveness to the infant's focus of attention and interest. Inter-rater agreement for the contingency of maternal responses in these two categories (Maternal responsiveness to change in infant's direction of gaze and Maternal responsiveness to infant's object-directed action) was $\kappa=.80$.

Mothers' responses to infants' vocalisations emerged as the third category of mind-mindedness. Some mothers responded to vocalisations by imitating the precise sound that the infant had produced, and a category of Imitation was therefore established. Imitation was included as a measure of mindmindedness since we reasoned that mothers would only imitate their infants if they interpreted their behaviour as having meaning and having been performed intentionally. In order to control for differences in the frequency of infant vocalisations, mothers' Imitation scores were expressed as a proportion of the total number of infant vocalisations.

These three categories of mind-mindedness (Maternal responsiveness to change in infant's direction of gaze; Maternal responsiveness to infant's object-directed action; and Imitation) thus arose from mothers' responses to their infants' behaviour.

The fourth category of mind-mindedness centred on mothers' elicitations of certain types of behaviour. Some mothers encouraged their infants to perform actions by themselves, such as retrieving an object that was out of reach, sitting up, manipulating a toy independently, and so on. Encouragement of autonomy was included as a measure of mind-mindedness since only mothers who deemed their infants to be capable of intentional action would encourage them to do things autonomously. In order to control for variations in mothers' verbosity and physical interaction during the session, mothers' Encouragement of autonomy scores were computed as a proportion of the total number of maternal behaviours coded.

The final category of mind-mindedness emerged not from assessing maternal responsiveness, or the function of mothers' behaviour, but from analysing the content of maternal speech. Of particular interest for the assessment of maternal mindmindedness were comments mothers made which appeared to relate to their infants' minds. Mothers' mind-related comments can be subdivided as follows. First, mothers commented on their infants' mental states, such as their knowledge, thoughts, desires, and interests. The following are some examples: "You know what that is, it's a ball"; "Which toy do you prefer?"; "I think that you think it's a drum". Second, mothers commented on their infants" mental processes, e.g. "Do you remember seeing a camel?"; "Do you recognise that?"; "Are you thinking?" Third, mothers refered to the infant's level of emotional engagement, including assertions that the infant was bored, worried, self-conscious, solemn, impressed, or excited. Fourth, the most sophisticated psychological states attributed to infants were instances where mothers commented on their infants' attempts to manipulate other people's beliefs, e.g. "You're joking"; "You're just teasing me"; "Are you playing games with me?". Finally, some mind-related comments involved the mother "putting words into her infant's mouth", so that the mother's discourse took on the structure of a dialogue between her infant and herself. Invariably, these comments detailed what the infant would be likely to say if he or she could speak: "She says, 'I'm not interested in him, I've already got one "'; "He says, 'I think I've got the hang of that now",; "Ah, they look nice', he says, 'them toys, cor they look lovely" "; "She says, 'Mummy, roll me back over'"; "Say, 'Mummy, can I play with something else?'”.

It should be noted that behaviours classified as mind-related comments were independent of the quality of interaction between mother and infant, and appeared to be solely a reflection of the mother's proclivity to use language to frame the interaction in a mentalistic context. This distinction is best illustrated using a real example. An interaction typical of many dyads consisted of the mother showing her infant his or her reflection in a mirror, which was included in the selection of toys. The less mind-minded mother's discourse accompanying this interaction was as follows: "Who's that in there? Is that you?"; whereas the more mind-minded mother framed the interaction thus: "Who do you think that is? Who do you think it is? Do you think that might be you?" Thus, two mothers who may score identically on this interaction in terms of general sensitivity and responsiveness may still demonstrate wideranging differences in their proclivity to talk about their infants' minds. What is therefore most interesting is that mothers ' mind-related comments were not determined by general maternal sensitivity or responsiveness, nor by the contiguity between infant behaviour and maternal response. However, since this category is not dependent upon the child's behaviour, and involves the mother inferring her infant's mental state, it was necessary to obtain an independent assessment of whether mothers' mind-related comments were accurate and appropriate.

Appropriateness of mothers' mind-related comments. Each mind-related comment was coded dichotomously as appropriate or inappropriate. The criteria for a comment being appropriate were as follows: (1) the independent coder agreed with the mother's reading of her infant's psychological state, e.g. if a mother commented that her infant wanted a particular toy, then it would be classified as an appropriate mental state comment if the independent coder concurred that the infant's behaviour was consistent with such a desire; (2) the comment linked current activity with similar events in the past or future, e.g. "Do you remember seeing a camel?" (while playing with a toy camel); (3) the comment served to clarify how to proceed if there was a lull in the interaction, e.g. "Do you want to look at the posters?" (after the infant had been gazing around the room and not focused on any object or activity for 5 seconds). Mindrelated comments were classified as inappropriate if: (1) the independent coder believed that the mother was misinterpreting her infant's psychological state, e.g. stating that the infant was bored with a toy when he/she was still actively engaged in playing with it; (2) the comment referred to a past or future event that had no obvious relation to current activity; (3) the mother asked what the infant wanted to do, or commented that the infant wanted or preferred a different object or activity, when the infant was already actively engaged in an activity or was showing a clear preference for a particular object; (4) the referent of the mother's comment was not clear, e.g. saying "You like that" when the object or activity to which the comment referred was not obvious.

A researcher who was blind to all other measures coded the videotapes to assess the appropriateness of mothers' mindrelated comments, and a second blind researcher coded a random fifth of the tapes. Inter-rater agreement was $\kappa=.79$.

In order to control for variations in mothers' verbosity during the session, mothers' Appropriate mind-related comments 
were computed as a proportion of the total number of maternal verbal comments coded during the testing session ${ }^{3}$.

\section{Phase Two Testing (Age 12 Months)}

Mothers were contacted by telephone 6 months after Phase One testing had been carried out and invited to come to the University for the second testing session, which was conducted when the infants were 12 months of age. The session began with the experimenter administering the "mental scale" from the Bayley Scales of Infant Development (Bayley, 1993), which is a standardised scale for assessing infants' general cognitive ability. The mean standardised score on the Bayley mental scale for the whole sample of infants was $M=91.70(S D=8.81)$.

After a short break, infant-mother security of attachment was assessed using the Strange Situation procedure (Ainsworth \& Wittig, 1969). The Bayley mental scale always preceded the Strange Situation procedure to control for possible effects of separation distress on the child's cognitive performance. The mental scale and Strange Situation were performed in different rooms.

Assessment of security of attachment. Forty-nine infants were classified as securely attached, and 22 as insecurely attached (14 insecure-avoidant, 5 insecure-resistant, and 3 insecure-disorganised). This distribution amongst the secure and insecure categories is similar to that reported by other researchers working on comparable samples of infants and mothers (e.g. Fish \& Stifter, 1995). The Strange Situation tapes were coded by the first author, who has formal training in the Strange Situation coding procedure (Ainsworth et al., 1978), and 16 of the tapes were chosen at random and coded for a second time by an independent trained rater. Both of the raters were blind to all of the other measures. Inter-rater agreement was $\kappa=.87$ using the ABCD categories, and $\kappa=.85$ using a secure versus insecure distinction. The classification of the one child about whom the raters disagreed was resolved by discussion.

\section{Results}

The six infant-mother pairs who had been used to establish the mind-mindedness criteria were excluded from the analysis, giving an overall sample size of 65 (33 boys, 32 girls). Of the excluded children, four were subsequently classified as securely attached, with the remaining two being insecure-avoidant.

\section{The Relation between Maternal Sensitivity and Mind-mindedness}

Our first aim was to investigate whether maternal sensitivity and mind-mindedness can be distinguished empirically, and how these constructs relate to one another. Table 1 shows the correlation matrix for all of the continuous variables. As Table 1 shows, the correlations between maternal sensitivity and the proportional scores for the mind-mindedness categories were all

\footnotetext{
${ }^{3}$ It could be argued that Appropriate mind-related comments should be calculated as a proportion of the total number of mind-related comments, rather than as a proportion of the overall total of maternal comments. We used this more conservative index in order to present a truer picture of the frequency with which mothers made appropriate mind-related comments throughout the testing session. We reasoned that a proportional score of 1.0 awarded to a mother who made only one (appropriate) mind-related comment might not provide an accurate picture of mind-minded discourse. That said, analyses using this alternative index produced exactly the same pattern of results as those reported in the Results section.
}

positive, and all except the relation between Sensitivity and Encouragement of autonomy were statistically significant. That said, the two mind-mindedness variables most strongly related to maternal sensitivity (Maternal responsiveness to change in infant's direction of gaze and Appropriate mind-related comments) each accounted for only $16 \%\left(r^{2}=.16\right)$ of the variance in sensitivity, suggesting that mind-mindedness and sensitivity are measuring related but distinct aspects of infant-mother interaction.

The proportional scores for the five categories of mindmindedness were positively correlated with one another, with the majority of these correlations reaching statistical significance. For the most strongly related pair, Maternal responsiveness to change in infant's direction of gaze accounted for $23 \%\left(r^{2}=.23\right)$ of the variance in Maternal responsiveness to infant's object-directed action, suggesting that the five categories of mind-mindedness are measuring related but distinct aspects of a mother's proclivity to treat her infant as an individual with a mind.

\section{Relations with Infant- and Mother-centred Variables}

Table 1 also shows the correlation coefficients for the relations between the infant-mother interaction variables (maternal sensitivity and maternal mind-mindedness), mothers' educational level, and infants' Bayley Scale scores. Maternal educational level was positively correlated with Maternal responsiveness to change in infant's direction of gaze, with more highly educated mothers being more likely to respond to changes in their infants' direction of gaze. Maternal educational level was not significantly correlated with any of the other infantmother interaction variables or with infants' general cognitive ability scores. Infants' Bayley Scale scores were not related to maternal sensitivity or to any of the mindmindedness variables.

Table 2 shows the mean scores of the secure and insecure groups with respect to infants' scores on the Bayley mental scale, and the three indices of infant behaviour from the infant-mother interactions at 6 months. As Table 2 shows, security of attachment was not related to any of these variables. Thus, secure and insecure group infants did not differ in their general cognitive ability or in their frequency of vocalisation, change in gaze, and object-directed activity during the 20 minute session. Table 2 shows the mean scores of the secure and insecure group mothers with respect to highest educational level and total number of comments made during the session. Mothers of securely and insecurely attached children did not differ in the level to which they had been educated or in how frequently they spoke during the testing session. Table 2 also shows the effect sizes for these relations. The effect size was calculated using the formula $d=\left(M_{\text {higher }}-M_{\text {lower }}\right) / S D$ (Cohen, 1977); two values are thus given (obtained by dividing by the two $S D$ s), representing the range of effect size. All of the effect sizes in Table 2 are small (Cohen, 1977), showing that the relation between attachment security and these variables is weak.

\section{Security-related Differences in Infant-Mother Interaction at 6 Months}

The mean scores for sensitivity and the mean proportional scores for the five maternal mind-mindedness 
Table 1

Correlation Matrix for the Continuous Variables

\begin{tabular}{lcccrrrrr}
\hline & MS & Gaze & Object & Imit & Aut & App & Mat Ed & Bayley \\
\hline MS & 1.00 & & & & & & & \\
Gaze & $.32^{* *}$ & 1.00 & & & & & & \\
Object & $.40^{* *}$ & $.48^{* *}$ & 1.00 & & & & & \\
Imit & $.30^{*}$ & $.26^{*}$ & .23 & 1.00 & & & & \\
Aut & .09 & $.36^{* *}$ & .21 & .19 & 1.00 & & & \\
App & $.40^{* *}$ & $.39^{* *}$ & $.40^{* *}$ & .18 & -.03 & 1.00 & & \\
MatEd & .22 & $.33^{* *}$ & .07 & -.06 & -.15 & .20 & 1.00 & \\
Bayley & -.02 & .21 & -.08 & .04 & .21 & .07 & -.05 & 1.00
\end{tabular}

MS = maternal sensitivity; Gaze = maternal responsiveness to change in infant's direction of gaze; Object $=$ maternal responsiveness to infant's object-directed action; Imit $=$ mother imitates infant's vocalisation; Aut $=$ mother encourages autonomy; App $=$ mothers' appropriate mindrelated comments; MatEd $=$ mothers' highest educational level; Bayley $=$ infants' Bayley Scale scores.

$* p<.025 ; * *<<.005$. Probability levels for any correlations using the variables MatEd and Bayley are two-tailed; the levels for all of the other correlations are one-tailed.

Table 2

Mean Scores for the Secure and Insecure Groups with Respect to Infant-and Mothercentred Variables

\begin{tabular}{|c|c|c|c|c|c|c|}
\hline \multirow[b]{3}{*}{ Variable } & \multicolumn{4}{|c|}{ Security of attachment } & \multirow{3}{*}{$\begin{array}{l}\text { Statistic } \\
t \text { values }^{\mathrm{a}}\end{array}$} & \multirow{3}{*}{$\begin{array}{c}\text { Effect sizes } \\
d\end{array}$} \\
\hline & \multicolumn{2}{|c|}{ Secure $(N=45)$} & \multicolumn{2}{|c|}{ Insecure $(N=20)$} & & \\
\hline & Mean & $S D$ & Mean & $S D$ & & \\
\hline Bayley & 92.23 & 8.77 & 89.55 & 9.17 & 1.14 & $0.29-0.31$ \\
\hline Vocalisation & 28.84 & 19.56 & 24.25 & 19.14 & 0.88 & $0.23-0.24$ \\
\hline Infant gaze & 73.24 & 22.32 & 71.95 & 19.00 & 0.23 & $0.06-0.12$ \\
\hline Infant object & 50.56 & 20.83 & 53.25 & 21.85 & 0.47 & $0.12-0.13$ \\
\hline Education & 2.60 & 1.72 & 2.90 & 1.25 & 0.70 & $0.17-0.24$ \\
\hline Comments & 141.71 & 41.78 & 130.35 & 43.74 & 1.00 & $0.26-0.27$ \\
\hline
\end{tabular}

Bayley $=$ infant score on the Bayley mental scale; Vocalisation $=$ total number of infant vocalisations; Infant gaze $=$ total number of changes in infant direction of gaze; Infant object $=$ total number of infant object-directed actions; Education = mothers' highest educational level; Comments $=$ total number of maternal comments.

a The $t$-tests were two-tailed; none reached statistical significance.

Table 3

Mean Scores and Effect Sizes for the Relation between Security of Attachment and Maternal Sensitivity, and Mean Proportional Scores and Effect Sizes for Relations between Security of Attachment and the Maternal Mind-mindedness Categories

\begin{tabular}{|c|c|c|c|c|c|c|}
\hline \multirow[b]{3}{*}{ Variable } & \multicolumn{4}{|c|}{ Security of attachment } & \multirow{3}{*}{$\begin{array}{l}\text { Statistic } \\
t \text { values }\end{array}$} & \multirow{3}{*}{$\begin{array}{c}\text { Effect sizes } \\
d\end{array}$} \\
\hline & \multicolumn{2}{|c|}{ Secure $(N=45)$} & \multicolumn{2}{|c|}{ Insecure $(N=20)$} & & \\
\hline & Mean & $S D$ & Mean & $S D$ & & \\
\hline Sensitivity & 5.80 & 2.02 & 4.50 & 2.26 & $2.31 *$ & $0.57-0.64$ \\
\hline Gaze & 0.49 & 0.14 & 0.46 & 0.21 & 0.71 & $0.14-0.21$ \\
\hline Object & 0.91 & 0.09 & 0.84 & 0.21 & $1.92 *$ & $0.33-0.78$ \\
\hline Imitate & 0.07 & 0.05 & 0.06 & 0.04 & 1.14 & $0.26-0.35$ \\
\hline Autonomy & 0.06 & 0.04 & 0.05 & 0.04 & 0.44 & $0.21-0.24$ \\
\hline App Mind & 0.11 & 0.06 & 0.05 & 0.04 & $4.34 * *$ & $1.00-1.50$ \\
\hline
\end{tabular}

Sensitivity = maternal sensitivity; Gaze = maternal responsiveness to change in the infant's line of gaze; Object $=$ maternal responsiveness to infant's object-directed action; Imitate $=$ mother imitates infant's vocalisation; Autonomy = mother encourages an autonomous act; App Mind = mothers' appropriate mind-related comments.

$* p<.025 ; * * p<.001$. Levels of significance are for one-tailed tests.

categories of the secure and insecure groups are given in Table 3. As this table shows, mothers who scored more highly on Ainsworth et al.'s (1971) scale of maternal sensitivity were more likely to have securely attached children. With respect to mind-mindedness and security of attachment, the relations between the proportional scores for all of the mind-mindedness categories and security were in the predicted direction, with mothers 
Table 4

Mean Scores for the Four Attachment Categories with Respect to Maternal Sensitivity and Mothers' Appropriate Mind-related Comments

\begin{tabular}{lccccc}
\hline & \multicolumn{2}{l}{ Maternal sensitivity } & & \multicolumn{2}{c}{ Appropriate mind-related comments } \\
\cline { 2 - 3 } \cline { 5 - 6 } Attachment category & Mean & $S D$ & & Mean & $S D$ \\
\hline Insecure-avoidant $(N=12)$ & 4.17 & 2.37 & & 0.04 & 0.04 \\
Secure $(N=45)$ & 5.80 & 2.02 & & 0.11 & 0.06 \\
Insecure-resistant $(N=5)$ & 6.00 & 1.87 & & 0.06 & 0.04 \\
Insecure-disorganised $(N=3)$ & 3.33 & 1.53 & & 0.05 & 0.02 \\
\hline
\end{tabular}

who scored more highly in these categories being more likely to have securely attached infants. Security of attachment was significantly related to Maternal responsiveness to infant's object-directed action and mothers' Appropriate mind-related comments. The relations between security of attachment and the categories Maternal responsiveness to change in infant's direction of gaze, Imitation, and Encouragement of autonomy were not statistically significant.

Table 3 also shows the effect sizes for these relations, calculated according to the formula given above. The effect size for the relation between Maternal sensitivity and security of attachment at 12 months was medium, that for the relation between Maternal responsiveness to infant's object-directed action and security was medium to large, and that between Appropriate mind-related comments and security was large (Cohen, 1977). Effect sizes for all of the other relations shown in Table 3 were small (Cohen, 1977).

\section{Mind-mindedness as a Predictor of Security of Attachment}

In order to determine the relative predictive strengths of the five mind-mindedness categories and subsequent security of attachment, a forward logistic regression was carried out. The five categories (Maternal responsiveness to change in infant's direction of gaze; Maternal responsiveness to infant's object-directed action; Imitation; Encouragement of autonomy; and Appropriate mindrelated comments) were the only factors entered into the regression. Appropriate mind-related comments was found to be the only predictor of security of attachment, $\chi^{2}$ $(N=65)=23.56, p<.001$.

\section{Overall Predictors of Security of Attachment}

Our next aim was to investigate the relative predictive strength of maternal sensitivity and mind-mindedness with respect to subsequent security of attachment. Given that mothers' Appropriate mind-related comments was the only mind-mindedness category found to be a predictor of attachment security, the other four mind-mindedness categories were omitted from the regression. Four factors were entered into a hierarchical logistic regression in the following order: Maternal educational level, Bayley Scale score, Maternal sensitivity, and Appropriate mind-related comments. When entered on the first step of the regression, Maternal educational level was not a predictor of attachment security, $\chi^{2}(N=65)=0.06$, n.s.; on the second step, Bayley Scale score was not a predictor of attachment security, $\chi^{2}(N=65)=2.00$, n.s. With mothers' educational level and infants' Bayley Scale scores entered into the prediction equation, Maternal sensitivity was found to be a significant predictor of attachment security, $\chi^{2}(N=65)=8.30, p<.005$, accounting for $6.5 \%$ of its variance. When Appropriate mind-related comments was entered on the final step of the regression, it was found to be a significant predictor of attachment security, $\chi^{2}(N=65)=17.62, p<.001$, accounting for $12.7 \%$ of its variance. Thus even when maternal sensitivity had been accounted for, mothers' Appropriate mind-related comments was a significant predictor of infant-mother security of attachment.

\section{Exploratory Analyses Using the ABC Attachment Categories}

A final aim was to investigate potential differences in early infant-mother interaction between the separate insecure attachment categories. Given the small numbers of children in the insecure categories, only descriptive statistics are reported here. Table 4 shows the mean scores of the four attachment categories for the two maternal variables found to be significant predictors of attachment security (Maternal sensitivity and mothers' Appropriate mind-related comments). No further analyses were conducted using the insecure-disorganised group since no specific predictions were made regarding this group and it contained only three infants. Effect sizes to compare the scores of the secure, insecure-avoidant, and insecure-resistant categories were calculated as before. The effect size for the difference in Maternal sensitivity scores between the secure and insecure-avoidant groups was found to be medium to large $(d=0.69-0.81)$, and that between the insecure avoidant and insecure-resistant groups was large $(d=0.77-0.98)$. However, the secure and insecure-resistant groups could not be distinguished from one another in terms of maternal sensitivity, with the resistant group scoring marginally higher than the secure group, resulting in a small effect size $(d=$ $0.10-0.11$ ). All three attachment groups could be distinguished from one another with respect to their scores on mothers' Appropriate mind-related comments, with large effect sizes between the secure and insecure-avoidant groups $(d=1.15-1.82)$ and secure and insecure-resistant groups $(d=0.82-1.36)$. The effect size for the difference between the avoidant and resistant groups was medium $(d=0.53-0.55)$.

\section{Discussion}

The initial goal of the study reported here was to establish empirical measures of mothers' proclivity to treat their 6-month-old infants as individuals with minds (maternal mind-mindedness) within a play context. Five 
measures of maternal mind-mindedness were devised on the basis of distinct ways in which mothers demonstrated their tendency to attribute intention to their infants. The first three of these measures dealt with mothers' responses to their infants' behaviour. The categories Maternal responsiveness to change in infant's direction of gaze and Maternal responsiveness to infant's object-directed action arose from maternal responses to infant actions that could be interpreted as cues for engagement in or disengagement from activities. Mothers' responses to their infants' changes in gaze or object-directed activity are indicative of mind-mindedness since they assess the mother's capacity to recognise that her infant's agenda may be different from her own. More mind-minded mothers will respond to their infants' change of attention, resulting in interactions that are contingent and responsive. Mothers also demonstrated their mind-mindedness by imitating their infants' vocalisations. Imitation was deemed to be indicative of mind-mindedness since mothers will only imitate their infants' vocalisations if they interpret them as having meaning and as having been performed intentionally. The fourth way in which mothers demonstrated mind-mindedness was by encouraging their infants to perform actions by themselves. Encouragement of autonomy was regarded to be a measure of mind-mindedness since only mothers who deem their infants capable of intentional action will encourage them to do things autonomously. The final mind-mindedness category focused on mothers' use of psychological discourse to refer to their infants' mental and emotional states and cognitive processes. In order to be coded as engaging in mind-minded behaviour, mothers did not only have to talk about their infants' minds, but had to make comments on mental states and processes that were deemed by independent raters to be accurate and appropriate. The category Appropriate mind-related comments was taken to be a measure of mothers' ability to read their infants' minds.

The first specific aim of the study reported here was to investigate the relation between maternal mind-mindedness and Ainsworth et al.'s (1971) original construct of maternal sensitivity. Our findings suggest that it is possible to differentiate between sensitive (as conceived by Ainsworth et al., 1971) and mind-minded maternal behaviours in interactions between mothers and their 6month-olds. Although each of the five mind-mindedness categories was positively correlated with maternal sensitivity, the mind-mindedness categories most strongly related to sensitivity (Maternal responsiveness to change in infant's direction of gaze and Appropriate mind-related comments) each only accounted for $16 \%$ of its variance. One can therefore argue that mind-mindedness and sensitivity are capturing related but distinct aspects of maternal behaviour, and that any relations that obtain between mind-mindedness and subsequent attachment security cannot be explained simply in terms of equivalence between mind-mindedness and sensitivity.

Our second aim was to attempt to replicate the finding that more sensitive mothers are more likely to establish secure attachment relationships subsequently with their infants. This aim was achieved. The medium effect size (Cohen, 1977) for the relation between these variables was in line with previous work, being very similar to the value reported in De Wolff and Van IJzendoorn's (1997) meta-analysis.

The third aim of the study was to test the hypothesis that infants whose mothers showed higher levels of mind- mindedness at 6 months will have formed secure attachment relationships with their infants 6 months later. Only two of the five mind-mindedness categories were significantly related to later security of attachment. Mothers who at 6 months commented appropriately on their infants' psychological states and processes, and who responded to their infants' object-directed activity, were more likely to have securely attached infants. However, an initial regression analysis showed that, of the five mind-mindedness variables, mothers' Appropriate mindrelated comments was the only significant predictor of attachment security. The hypothesised relation between early maternal mind-mindedness and subsequent security was thus not supported for all of the mind-mindedness categories.

The fourth aim was to investigate the relative strength of maternal sensitivity and maternal mind-mindedness as predictors of attachment security. A hierarchical regression showed that both maternal sensitivity and mothers' Appropriate mind-related comments were significant predictors of subsequent security. After maternal sensitivity had been entered into the regression (accounting for $6.5 \%$ of the variance), mothers' appropriate mind-related comments accounted for a further $12.7 \%$ of the variance in attachment security. This category of maternal mind-mindedness was therefore a better predictor of infant-mother attachment security than maternal sensitivity.

Our final aim was to explore whether differences in maternal mind-mindedness could distinguish between infants across the three major attachment categories. Although the data from the insecure-avoidant and insecure-resistant groups should be treated with caution due to the low numbers of infants in these groups, there appears to be reason to suggest that differences in mothers' appropriate mind-related comments can discriminate between different types of insecurity. The insecure-avoidant group mothers made fewer appropriate mind-related comments than mothers in the insecure-resistant group. Mothers in both of the insecure groups made significantly fewer appropriate mind-related comments than their counterparts in the secure group. In contrast, there was no significant difference in sensitivity scores between the secure and resistant group mothers; indeed, the resistant group mothers scored marginally higher on this measure than their secure group counterparts. Thus, in this sample of infants, mothers' appropriate mind-related comments appeared better able than maternal sensitivity to distinguish between infants across the three attachment categories.

How can one explain the finding that mothers' proclivity to comment accurately on their infants' minds predicts security of attachment even after differences in maternal sensitivity have been accounted for? One possible explanation is that the construct of maternal mind-mindedness takes into account the appropriateness of mothers' interactions with their infants, whereas the global scale of maternal sensitivity does not distinguish recognition of the infant's needs (suggested by Ainsworth et al. to be shared by both secure and insecure group mothers) from appropriate response to such needs. Thus, the problem with maternal sensitivity as a predictor of attachment security lies not in its original definition, but in the way it has come to be operationalised.

An important key to explaining this finding may lie in the fact that the present study's measure of Appropriate mind-related comments is an index of the mother's 
capacity accurately to represent the mental and emotional states of her infant. As such, this capacity can be seen to relate to the processes involved in the formation and operation of internal working models (IWM: Bowlby, 1973, 1980) of attachment. Although one's IWM of an attachment figure is constructed on the basis of one's experiences with that individual, the model is representational, since it enables one to predict the form of future interactions with the attachment figure: whether he or she will be accessible or unavailable, sensitive or intrusive, and so on. Indeed, a mother's tendency to frame interactions in terms of her infant's desires, intentions, beliefs, and emotions may provide us with a naturalistic measure of the mother's internal working model of self with child, just as the Adult Attachment Interview (AAI: George, Kaplan, \& Main, 1985) provides a measure of one's IWM of self with parent. In contrast, maternal sensitivity is an index of behaviour with no representational component, and it therefore lacks clear theoretical links with important concepts such as the IWM.

This suggestion is borne out by the findings of researchers who have sought to establish how individual differences in parents' AAI classifications relate to their attachment relationships with their own children. For example, Fonagy, Steele, and Steele (1991) assessed parental IWMs of attachment prenatally in a primiparous sample, and demonstrated an impressive level of concordance between a mother's secure classification on the AAI and a secure attachment relationship with her child at 12 months. In subsequent work, Fonagy and colleagues (e.g. Fonagy, Steele, Steele, Moran, \& Higgitt, 1991; Fonagy et al., 1994) have investigated this relation further by developing the "reflective-self scale" which is used to assess AAI transcripts for interviewees' awareness of their own and other people's mental processes and functioning. They reported that reflective-self function was the underlying variable accounting for the AAI's predictive power with respect to infant-mother security of attachment. Thus, mothers who tended to invoke mental states when explaining the behaviour of others during the AAI were more likely to have securely attached children. There is an obvious parallel between a mother's mentalistic comments during the AAI and her mindrelated comments during interactions with her infant at 6 months, and it is thus perhaps unsurprising that both are related to subsequent security of attachment. Indeed, individual differences in maternal mind-mindedness may help to bridge what Van IJzendoorn (1995) referred to as the "transmission gap" between parents" mental representations of childhood attachment experiences and the formation of secure attachment relationships with their own children. That is, while AAI classification and parental sensitivity have both been found to be independent predictors of attachment security, there is no strong relation between a secure AAI classification and greater parental sensitivity. Sensitive caregiving behaviour cannot therefore account for the variance shared between AAI classification and infant security of attachment.

How might the construct of maternal mind-mindedness help us to bridge this "transmission gap"? One answer is that, in contrast to measures based purely on differences in maternal behaviour (e.g. maternal sensitivity), our construct of mind-mindedness (and in particular the discourse-based category of Appropriate mind-related comments) is a measure of the mother's representation of her infant's mental states. As Meins (1999) has argued, this representational component makes it considerably easier to see how mothers' behaviour is in turn determined by their representations of their own attachment relationships (as assessed by, for example, the AAI). At the same time, our measure could be argued to be an advance on previous interview-based assessments of parental representations (e.g. Fonagy et al., 1994; Meins et al., 1998), because it shows how such representations are manifested in the language mothers use in real-life interactions with their infants.

As well as having conceptual and theoretical links with other predictors of attachment, the observed relation between a mother's tendency to comment accurately on her infant's mind and subsequent security of attachment may also relate to recent findings about the consequences of a secure attachment relationship. For example, the secure-group mother's "mentalisation" of her infant might shed light on the finding that securely attached children outperform their insecurely attached peers on standard "theory of mind" tasks (Fonagy, Redfern, \& Charman, 1997; Meins et al., 1998). Such mentalisation can be presumed to involve, inter alia, exposing infants to mental-state language from an early age, which may encourage children to understand themselves and others as mental agents, and thus lead to precocity in the awareness of the relation between one's beliefs and one's behaviour. Although this relation has yet to be tested empirically, Meins et al.'s results and the findings reported here suggest that there may be long-term continuity in secure-group mothers' proclivity to regard their children as mental agents. The greater mindmindedness of secure-group mothers may thus have influences which extend beyond the formation of the attachment relationship, and may account for certain security-related differences in children's later development. Indeed, Meins and Fernyhough (1999) argued that the concept of mind-mindedness may help to explain why other social factors, such as individual differences in family structure (Lewis, Freeman, Kyriakidou, Maridaki-Kassotaki, \& Berridge, 1996; Ruffman, Perner, Naito, Parkin, \& Clements, 1998) and familial discourse about emotions and causal relations (Dunn, Brown, Slomkowski, Tesla, \& Youngblade, 1991) influence theory of mind development. For example, other family members are also likely to demonstrate mind-mindedness in their interactions with young children, and by talking about feelings and how people's behaviour impacts on the lives of others, families are implicitly treating their children as individuals with minds, capable of understanding complex relationships and events.

Much work remains to be done in continuing to develop the construct of mind-mindedness and in establishing its connections with both maternal sensitivity and attachment security. Given our focus on observational context, it is important to investigate mind-mindedness in other situations, such as interactions in the home where the mother will be engaged in caregiving as well as play activities. If mind-mindedness is to become established as a reliable predictor of attachment security, it is necessary to demonstrate that mind-minded discourse also accompanies activities more commonly associated with the formation of attachment relationships. For example, mothers' development of differential responses to early crying may depend upon their willingness or ability to interpret crying as a means of communication. Similarly, it is important to investigate whether individual differences in maternal mind-mindedness predict attach- 
ment security in other cultures and in mothers of differing socioeconomic status, since maternal sensitivity has been found to relate to security across wide-ranging populations (Egeland \& Farber, 1984; Goldberg et al., 1986; Grossmann et al., 1985).

That said, the present finding that mothers' use of appropriate mind-related comments is a better predictor of attachment security than maternal sensitivity marks an important development in our understanding of the antecedents and consequences of individual differences in the attachment relationship. Whereas our rethinking of the concept of maternal sensitivity has maintained conceptual and theoretical links with established predictors of attachment security, the study reported here shows how security might be influenced by mothers' representations of their infants' mental states, as manifested in the language they use in real-life play interactions. The concept of maternal mind-mindedness thus has the potential to explain the poorly understood links between a mother's tendency to talk coherently about her own attachment relationships during the AAI and her ability subsequently to form a secure attachment relationship with her child. Our findings therefore address Thompson's (1997) analysis of the needs of future attachment research: "Understanding why sensitive responsiveness contributes to a secure attachment, and how this is associated with later working models of self and relationships, may be the most important theoretical problem for attachment researchers in the years to come" (p. 597).

Acknowledgements - The research reported in this paper was supported by two research grants from Staffordshire University. We thank Paul Harris, Sue Leekam, Anne Campbell, Joanne Martin, Bronia Hurst, and the three anonymous reviewers for their invaluable contributions to this paper.

\section{References}

Ainsworth, M. D. S., Bell, S. M., \& Stayton, D. J. (1971). Individual differences in Strange Situation behavior of one year olds. In H. R. Schaffer (Ed.), The origins of human social relations. New York: Academic Press.

Ainsworth, M. D. S., Bell, S. M., \& Stayton, D. J. (1974). Infant-mother attachment and social development: Socialisation as a product of reciprocal responsiveness to signals. In M. P. M. Richards (Ed.), The introduction of the child into a social world. London: Cambridge University Press.

Ainsworth, M. D. S., Blehar, M. C., Waters, E., \& Wall, S. (1978). Patterns of attachment: Assessed in the strange situation and at home. Hillsdale, NJ: Lawrence Erlbaum.

Ainsworth, M. D. S., \& Wittig, B. A. (1969). Attachment and exploratory behavior of one year olds in a strange situation. In B. M. Foss (Ed.), Determinants of infant behaviour Vol. 4. New York: Barnes \& Noble.

Bayley, N. (1993). Bayley Scales of Infant Development (2nd ed.). San Antonio, TX: The Psychological Corporation, Harcourt Brace \& Company.

Beeghly, M., Bretherton, I., \& Mervis, C. (1986). Mothers' internal state language to toddlers: The socialisation of psychological understanding. British Journal of Developmental Psychology, 4, 247-260.

Belsky, J., Rovine, M., \& Taylor, D. G. (1984). The Pennsylvania Infant and Family Development Project, III: The origins of individual differences in infant-mother attachment: Maternal and infant contributions. Child Development, $55,718-728$

Bowlby, J. (1973). Attachment and loss: Vol. 2. Separation: Anxiety and anger. London: Hogarth Press.
Bowlby, J. (1980). Attachment and loss: Vol. 3. Loss sadness and depression. London: Hogarth Press.

Cohen, J. (1977). Statistical power analysis for the behavioural sciences (rev. ed.). New York: Academic Press.

De Wolff, M. S., \& Van IJzendoorn, M. H. (1997). Sensitivity and attachment: A meta-analysis on parental antecedents of infant attachment. Child Development, 68, 571-591.

Dunn, J., Bretherton, I., \& Munn, P. (1987). Conversations about feeling states between mothers and their young children. Developmental Psychology, 23, 132-139.

Dunn, J., Brown, J., Slomkowski, C., Tesla, C., \& Youngblade, L. M. (1991). Young children's understanding of other people's feelings and beliefs: Individual differences and their antecedents. Child Development, 62, 1352-1366.

Egeland, B., \& Farber, E. (1984). Infant-mother attachment: Factors related to its development and change over time. Child Development, 60, 753-771.

Fish, M., \& Stifter, C. A. (1995). Patterns of mother-infant interaction and attachment: A cluster-analytic approach. Infant Behavior and Development, 18, 435-446.

Fonagy, P., Redfern, S., \& Charman, A. (1997). The relationship between belief-desire reasoning and a projective measure of attachment security (SAT). British Journal of Developmental Psychology, 15, 51-63.

Fonagy, P., Steele, H., \& Steele, M. (1991). Maternal representations of attachment during pregnancy predict the organisation of infant-mother attachment at one year of age. Child Development, 62, 891-905.

Fonagy, P., Steele, M., Steele, H., Higgitt, A. C., \& Target, M. (1994). The Emmanuel Miller Memorial Lecture 1992: The theory and practice of resilience. Journal of Child Psychology and Psychiatry, 35, 231-257.

Fonagy, P., Steele, M., Steele, H., Moran, G. S., \& Higgitt, A. C. (1991). The capacity for understanding mental states: The reflective self in parent and child and its significance for security of attachment. Infant Mental Health Journal, 12, 201-218.

George, C., Kaplan, N., \& Main, M. (1985). The Berkeley Adult Attachment Interview. Unpublished protocol, Department of Psychology, University of California, Berkeley.

Goldberg, S., Perotta, M., Minde, K., \& Corter, C. (1986). Maternal behavior and attachment in low-birth-weight twins and singletons. Child Development, 57, 34-46.

Goldsmith, H. H., \& Alansky, J. (1987). Maternal and infant temperamental predictors of attachment: A meta-analytic review. Journal of Consulting and Clinical Psychology, 55, 805-816.

Grossmann, K., Grossmann, K. E., Spangler, G., Suess, G., \& Unzner, L. (1985). Maternal sensitivity and newborns' orientation responses as related to quality of attachment in northern Germany. In I. Bretherton \& E. Waters (Eds.), Growing points in attachment theory and research (pp. 233-268). Monographs of the Society for Research in Child Development, 50 (1-2, Serial No. 209).

Isabella, R. A. (1993). Origins of attachment: Maternal interactive behavior across the first year. Child Development, 64, 605-621.

Isabella, R. A., Belsky, J., \& Von Eye, A. (1989). Origins of infant-mother attachment: An examination of interactional synchrony during the infant's first year. Developmental Psychology, 25, 12-21.

Lewis, C., Freeman, N. H., Kyriakidou, C., MaridakiKassotaki, K., \& Berridge, D. M. (1996). Social influences on false belief access: Specific sibling influences or general apprenticeship? Child Development, 67, 2930-2947.

Main, M. (1981). Avoidance in the service of attachment; A working paper. In K. Immelman, G. Barlow, L. Petrinovich, \& M. Main (Eds.), Behavioral development (pp. 651-693). Cambridge: Cambridge University Press.

Meins, E. (1997). Security of attachment and the social development of cognition. Hove, U.K.: Psychology Press. 
Meins, E. (1998). The effects of security of attachment and maternal attribution of meaning on children's linguistic acquisitional style. Infant Behavior and Development, 21, 237-252.

Meins, E. (1999). Sensitivity, security and internal working models: Bridging the transmission gap. Attachment and Human Development, 1, 325-342.

Meins, E., \& Fernyhough, C. (1999). Linguistic acquisitional style and mentalising development: The role of maternal mind-mindedness. Cognitive Development, 14, 363-380.

Meins, E., Fernyhough, C., Russell, J., \& Clark-Carter, D. (1998). Security of attachment as a predictor of symbolic and mentalising abilities: A longitudinal study. Social Development, 7, 1-24.

Ruffman, T., Perner, J., Naito, M., Parkin, L., \& Clements,
W. A. (1998). Older (but not younger) siblings facilitate false belief understanding. Developmental Psychology, 34, 161-174.

Schölmerich, A., Fracasso, M. P., Lamb, M E., \& Broberg, A. G. (1995). Interactional harmony at 7 and 10 months of age predicts security of attachment as measured by Q-sort ratings. Social Development, 4, 62-74.

Thompson, R. A. (1997). Sensitivity and security: New questions to ponder. Child Development, 68, 595-597.

Van IJzendoorn, M. H. (1995). Adult attachment representations, parental responsiveness, and infant attachment: A meta-analysis on the predictive validity of the Adult Attachment Interview. Psychological Bulletin, 117, 387-403.

Manuscript accepted 16 January 2001 